\title{
The research on the influence of kinetic parameters on the vibration of blood pump in viscoelastic implantation environment
}

\author{
Zhong Yun, Chuang Xiang ${ }^{*}$ and Liang Wang \\ School of Mechanical and Electrical Engineering, Central South University, Changsha 410083, R.P. China
}

\begin{abstract}
The implantable axial flow blood pump would be vibrated when in actual use because of the viscoelastic implantation environment, unbalanced driven force, high speed rotor and so on. The blood pump researched in the paper was designed to be implanted in ascending aortic and driven by external magnetic force. The kinetic equation of blood pump worked in viscoelastic implantation environment was built, and then the blood pump's vibration influenced by viscoelasticity of implantation environment, running speed of blood pump, weight of blood pump were studied. Appropriate damping coefficient of implantation environment, running speed of blood pump and weight of blood pump for blood pump's more stable working were found in the paper. The paper could provide theoretical basis and research method for blood pump's structure parameters and work performance improving.
\end{abstract}

\section{Introduction}

Blood pump can take place the heart of heart patient temporary or long-term [1-4]. In recent years, most of the researches on blood pump focused on the impeller structure, flow channel, drive system, hemolysis, suspension mechanism and so on [5-9]. But the research on the kinetic system of blood pump in implantation environment was few. The implantable blood pump was often implanted in the chest, aortic valve and ascending aortic $[10,11]$. There were blood vessel, myocardium, connective tissue and other tissues around the blood pump. These tissues were all viscoelastic material [12, 13], would be deformed when being compressed or stretched. In order to be implanted easily, the smaller was the blood pump the better, so the speed of blood pump would be very high (about 10000r/min) [14,15] for supplying enough blood flow. The driven system of blood pump often was brushless direct-current motor [16]. The blood pump system of this paper was large gap external magnetic driven system. The driven force was changed periodically for providing periodical driven moment. The changed driven force and viscoelastic implantation environment would cause the blood pump vibration when working.

The blood pump researched in this paper was miniature implantable axial blood pump and the position of being implanted was ascending aortic. The paper modeled the implantation environment of blood pump, and then built the kinetic equation. Through solving the kinetic equation in SIMULINK, the vibration acceleration, velocity and displacement of blood pump influenced by implantation environment, rotor speed and weight of blood pump were researched. The research in this paper could provide theoretical evidence for the optimization of blood pump.

\section{Modelling and method}

The blood pump researched in this paper was implanted in ascending aortic. There were vessel, connective tissue and other tissues wrapped the blood pump. As shown in Fig.1, the implantation environment was modeled to be two coaxial cylinders, one represented the vessel wall and the other represented the myocardial tissue, connective tissue and other tissues, for simplifying the calculation. The vessel's inner diameter is $D_{1}=20 \mathrm{~mm}$ (blood pump's outer diameter), the thickness of vessel wall [13] is about $1 \mathrm{~mm}$ (vessel's outer diameter $D_{2}=22$ $\mathrm{mm}$ ), the tube's outer diameter is $D=40 \mathrm{~mm}$, the axial length is $L=80 \mathrm{~mm}$ (the length of the blood pump), the outer wall is fixed. All the material was regarded as isotropic [13]. Because the external driven force was perpendicular to the axis of blood pump, so the axial length of cylinder which was equal to the length of blood pump was considered in the paper.

*Corresponding author: $\underline{08105077 @ 163 . c o m}$ 


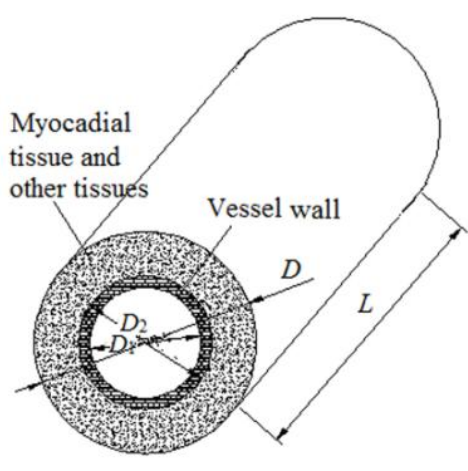

Fig. 1. The model of blood pump's implantation environment. $D$ was the outside diameter of cylinder which represented the myocardial tissue and other tissues. $D_{1}$ was the inside diameter of vessel wall and $D_{2}$ was the outside diameter. $L$ was the length of the cylinders.

In order to build the kinetic model of blood pump in implanted environment, the spring-damping model [13] was used to simulate the vessel and the tissues around vessel. According to the positional relationship between the ascending aortic vessel wall and tissue, the viscoelastic model of the implanted environment was established which was shown in Fig. 2.

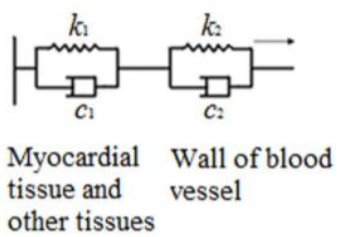

Fig. 2. Spring-damping model of blood pump's implantation environment. $c_{1}$ and $c_{2}$ were damping coefficient. $k_{1}$ and $k_{2}$ were elasticity coefficient.

The elasticity coefficient and damping coefficient of the implanted environment's viscoelastic model can be calculated by the formulas:

$$
\begin{gathered}
k=2 \frac{k_{1} k_{2}}{k_{1}+k_{2}} \\
c=2 \frac{c_{1} c_{2}}{c_{1}+c_{2}}
\end{gathered}
$$

Where, $k, k_{1}, k_{2}$ were elasticity coefficient of implantation environment, myocardial tissue and other tissues, blood vessel wall respectively. $c, c_{1}, c_{2}$ were the damping coefficient of implantation environment, myocardial tissue and other tissues, blood vessel wall respectively.

Substituting numerical value [17] into the Eq. (1) and Eq. (2), the elasticity coefficient of blood pump's implanted environment is $k=981.7 \mathrm{~N} / \mathrm{m}$, the damping coefficient of blood pump's implanted environment is $c=21.4 \mathrm{~N} \cdot \mathrm{s} / \mathrm{m}$.

Considered the driven force was perpendicular to the axis of blood pump, the kinetic model of blood pump working in viscoelastic implantation environment can be simplified to be two force components, two springdamping system and a mass point which were shown in Fig.3. The mass point represented the blood pump. Two force components represented the driven force divided into horizontal and vertical directions. Two springdamping systems represented the implantation environment's resistance to the blood pump's movement which was divided into horizontal and vertical directions. Because the material is isotropic, the parameters of two spring-damping systems were same, $c_{1}^{\prime}=c_{2}^{\prime}=c, k_{1}^{\prime}=k_{2}^{\prime}=k$.

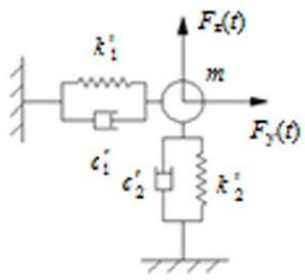

Fig. 3. The kinetic system of blood pump in implantation environment.

Based on the Newton's second law, the kinetic equation of blood pump working in viscoelastic implantation environment was:

$$
\left.\left.\left[\begin{array}{cc}
m & 0 \\
0 & m
\end{array}\right] \ddot{s}\right\}+\left[\begin{array}{cc}
c_{1}^{\prime} & 0 \\
0 & c_{2}^{\prime}
\end{array}\right] \dot{s}\right\}+\left[\begin{array}{cc}
k_{1}^{\prime} & 0 \\
0 & k_{2}^{\prime}
\end{array}\right]\{s\}=\left\{\begin{array}{c}
F_{y}(t) \\
F_{z}(t)
\end{array}\right\}
$$

$m$ was the weight of blood pump. $s=[y, z]^{\mathrm{T}}$, represented the displacement of blood pump, could be divided into horizontal direction $y$ and vertical direction $z$. The driven force was divided into horizontal direction $F_{y}(t)$ and vertical direction $F_{z}(t)$ which were shown in Fig.4 [18].

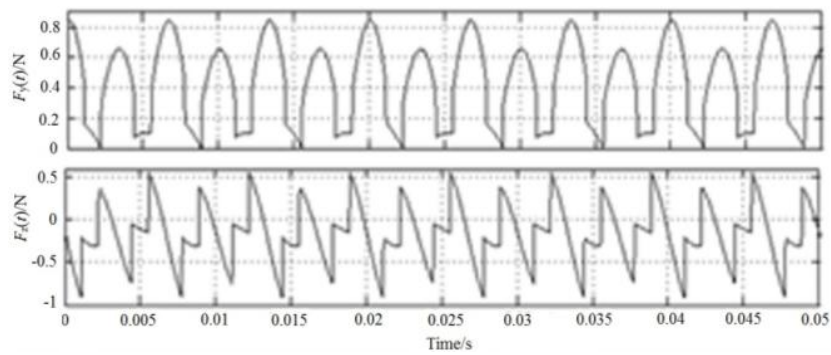

Fig. 4. The driven force which was divided into horizontal direction $F_{y}(t)$ and vertical direction $F_{z}(t)$.

The natural frequency of the damping system $\omega_{\mathrm{d}}$ can be calculated by the formula:

$$
\omega_{\mathrm{d}}=\sqrt{\left(1-\xi^{2}\right) \frac{k}{m}}
$$

In the Eq.(4), $\xi=c /\left(2(m k)^{1 / 2}\right)$. The kinetic system's natural frequency was calculated to be $f d 1=f d 2=\omega_{\mathrm{d}} /(2 \pi)$ $\approx 9.2 \mathrm{~Hz}$

\section{Results and discussion}

\subsection{Vibration response}

Modules in SIMULINK were used to solve the kinetic equation. Fig.5 showed the vibration response in the direction of $\mathrm{Y}$ and $\mathrm{Z}$. The blood pump's displacement increased rapidly in the initial stage. Then blood pump 
achieved the maximum displacement at about 0.06s, both in the direction of $\mathrm{Y}$ and $\mathrm{Z}$. The displacement fluctuation decreased gradually later. At last he blood pump reached a dynamic balance and vibrated periodically with tiny amplitude relative to the equilibrium position. The cycle of the balance vibration and exciting force are the same, while the equilibrium position in the direction of $\mathrm{Y}$ and $\mathrm{Z}$ were respectively $4.5 \times 10^{-3} \mathrm{~m}$ and $-1.6 \times 10^{-3} \mathrm{~m}$.
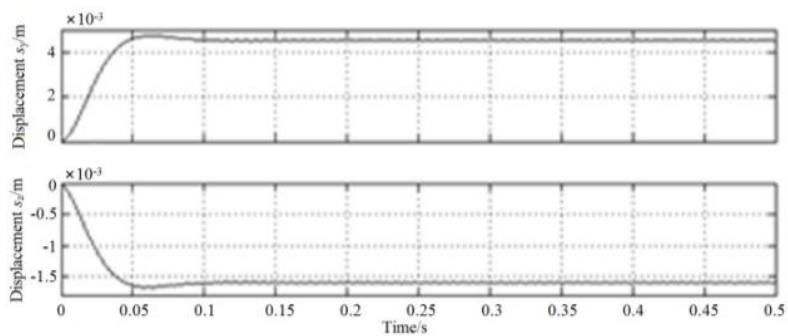

Fig. 5. Vibration responses of blood pump working in implantation environment. $S_{\mathrm{y}}$ and $S_{\mathrm{z}}$ were the vibration displacement in the direction of $\mathrm{Y}$ and $\mathrm{Z}$ respectively.

\subsection{Vibration influenced by implantation environment}

The parameters of implantation environment vary from person to person, and are different when the person in different ages. According solving the Eq.(3) when the damping coefficient varied from 1 to $30 \mathrm{~N} \cdot \mathrm{s} / \mathrm{m}$, the paper researched the blood pump's vibration acceleration $A_{a}$, velocity $A_{v}$ and displacement $A_{s}$ affected by damping coefficient which was shown in Fig.6. As shown in Fig.6, the $A_{a}, A_{v}$ and $A_{S}$ were all divided along horizontal and vertical directions into $A_{a y}, A_{a z}, A_{v y} A_{v z}, A_{s y} A_{s z}$ respectively. As the damping coefficient increasing, the vibration acceleration first decreased, then began to rise at about $8 \mathrm{~N} \cdot \mathrm{s} / \mathrm{m}$; The vibration velocity decreased all the time. When the damping coefficient was small, the amplitude was larger, and the change in the amplitude was also larger. But when the damping coefficient was bigger than $4 \mathrm{~N} \cdot \mathrm{s} / \mathrm{m}$, the change of damping coefficient had little effect to the vibration displacement.

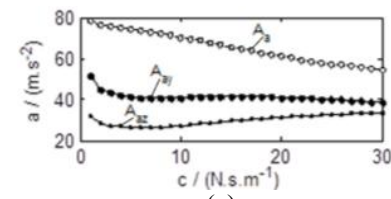

(a)

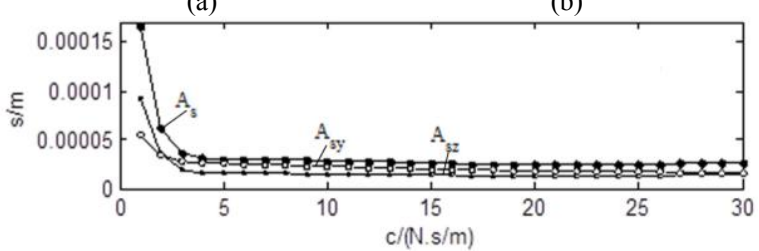

(c)
Fig. 6. The vibration acceleration $A_{a}(a)$, velocity $A_{v}(b)$ and displacement $A_{S}(c)$ affected by damping coefficient. $A_{a y}$ and $\mathrm{A}_{\mathrm{az}}$ were components of $\mathrm{A}_{\mathrm{a}}$. $\mathrm{A}_{\mathrm{vy}}$ and $\mathrm{A}_{\mathrm{vz}}$ were components of $A_{v} \cdot A_{s y}$ and $A_{s z}$ were components of $A_{s}$.

\subsection{Vibration influenced by rotor speed}

The speed of blood rotor would be change in order to supply different blood flow for person who was implanted the blood pump in different movement state. The rotor speed had direct relationship with the frequency of exciting force. So, the rotor speed was an important parameter for kinetic system of implantation environment. The vibration acceleration, velocity and displacement of blood pump influenced by rotor speed which varied from 1000 to $12000 \mathrm{r} / \mathrm{min}$ were researched in the paper. Fig. 7 showed the relationship between rotor speeds with vibration acceleration. When the rotor speed increased, the blood pump's vibration acceleration, velocity and displacement all increased at low speed, and then decreased. The affect of rotor speed to the blood pump's vibration acceleration, velocity and displacement was obvious at low speed ( $<4000 \mathrm{r} / \mathrm{min})$, small at middle speed (4000 8000 r/min), and not obvious at high speed (>8000 r/min). When the rotor speed at 1000-2000 r/min, the vibration amplitude of blood pump was large, the blood pump should reduce work time in this speed range. The blood pump had a good work performance when rotor speed was higher than $8000 \mathrm{r} / \mathrm{min}$.

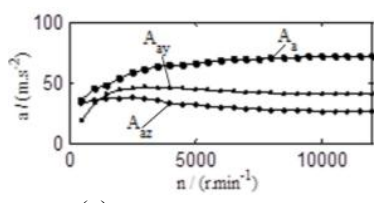

(a)

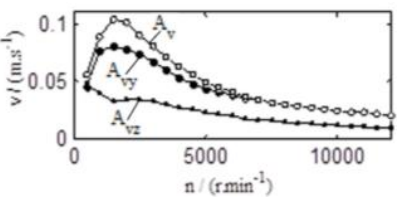

(b)

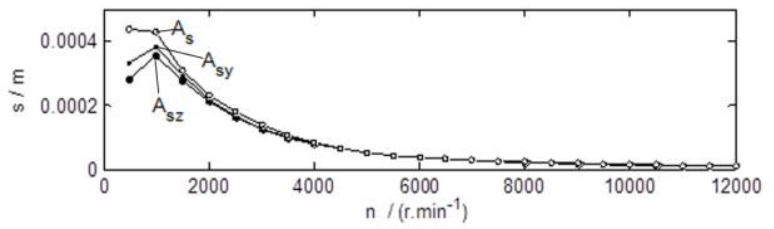

(c)

Fig. 7. The vibration acceleration Aa (a), velocity Av (b) and displacement As (c) affected by speed of blood pump's rotor. $A_{a y}$ and $A_{a z}$ were components of $A_{a} \cdot A_{v y}$ and $A_{v z}$ were components of $A_{v}$. $A_{s y}$ and $A_{s z}$ were components of $A_{s}$.

\subsection{Vibration Influenced by Blood Pump's Weight}

The weight of blood pump was a parameter in kinetic equation Eq.(3). The paper researched the vibration acceleration, velocity and displacement of blood pump influenced by blood pump's weight changing from 0.01 $\mathrm{Kg}$ to $0.04 \mathrm{Kg}$. The results were shown in Fig. 8. As the blood pump's weight increasing, the vibration acceleration, velocity and displacement of blood pump decreased, and the degree of decrease was smaller and smaller. It seemed that the heavier of the blood pump, the kinetic system of blood pump would be more stable. But the increase of blood pump's weight would lead more difficult implantation and worse comfortable. Form Fig. , we knew the weight of blood pump affected vibration displacement not obviously when the weight of blood pump was heavier than $0.03 \mathrm{Kg}$. So, it's better for the weight of blood pump be about $0.03 \mathrm{Kg}$. 


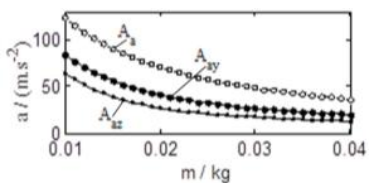

(a)

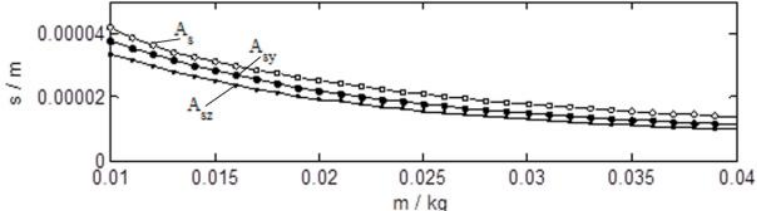

(c)

Fig. 8. The vibration acceleration $A_{a}(a)$, velocity $A_{v}(b)$ and displacement $A_{s}(c)$ influenced by weight of blood pump. $A_{a y}$ and $A_{a z}$ were components of $A_{a} \cdot A_{v y}$ and $A_{v z}$ were components of $A_{v} . A_{s y}$ and $A_{s z}$ were components of $A_{s}$.

\section{Conclusions}

The spring-damping model was used in the paper to simulate the viscoelasticity implantation environment of blood pump. By solving the kinetic equation of blood pump in implantation environment through SIMULINK, the vibration acceleration, velocity and displacement of blood pump influenced by implantation environment, rotor speed and weight of blood pump were researched. The research found the kinetic system of blood pump in viscoelasticity implantation environment would be more stable when the damping coefficient of implantation environment was larger than $4 \mathrm{~N} \bullet \mathrm{s} / \mathrm{m}$, the rotor speed of blood pump was higher than $8000 \mathrm{r} / \mathrm{min}$, and the weight of blood pump was about $0.03 \mathrm{Kg}$. In the research, the implantation environment was simplified and the driven force only considered. There were other complicated tissues around blood pump, fluid force, motion excitation and other factors should be considered in kinetic system of blood in implantation environment in future work.

This work was supported by the National Natural Science Foundation of China (Grant No. 31670999, 31271057, 51475477).

\section{References}

1. M. Abshire, C. R. Dennison, S D Russell, J. Card. Fail., 20, 12(2014), 973-983.

2. N. Moazami, K. Fukamachi, M. Kobayashi, N. G. Smedira, K. J. Hoercher, J. Heart Lung Transpl., 32, 1(2013), 1-11.

3. L. Bellumkonda, P. Bonde, International Anesthesiology Clinics, 50, 3(2012), 123-145.

4. S. P. Pinney, J. Card. Fail., 21, 10(2015), 825-834.

5. N. Moazami, R. J. Steffen, Y. Naka, U. Jorde, S. Bailey, S. Murali, Ann. Thorac. Surg., 98, 2(2014), 541-547.

6. H. Horiguchi, T. Tsukiya, T. Takemika, T. Nomoto, Y. Tsujimoto, International Journal of Fluid Machinery and Systems, 8, 1(2015), 1-12.
7. T. Murashige, R. Kosaka, M. Nishida, O. Maruyama, T. Yamane, K. Kuwana, Y. Kawaguchi, 35th Annual International Conference of the IEEE EMBS, (2013),2732-2735.

8. J. C. Wu, J. F. Antaki, J. Verkaik, S. snyder and M. Ricci, J. Fluid. Eng., 134, 4(2012),379-389.

9. S. Saito, K. Yamazaki, T. Nishinaka, Y. Ichihara, M. Ono, S. Kyo, J. Heart Lung Transpl., 33, 6(2014), 599-608.

10. E. J. Molina, S. W. Boyce, Semin. Thorac. Cardiovasc. Surg., 25, 1(2016), 56-63.

11. E. Okamoto, Y. Ishida, T. Yano, Y. Mitamura, J. Artif. Organs, 18, 2(2015), 181-184.

12. Q. Yu, Am. J. physiol., 265, (1993), 1052-1060.

13. Z. Tao, Biofluid Mechanics (Beijing, Science Press, 1984).

14. M. S. Slaughter, I. I. Michael, D. Tamez, T. Horrell, J. Graham, S. P. Patroklos, Tex. Heart I. J., 36, 1(2009), 12.

15. G. R. Li, X. D. Zhu, B. S. Tian, Z. C. Hao, H. F. Cheng, China Medical Device Information, 6, (2013), 20-24.

16. B. Pratondo, I. Andi, R. A. Akbar and F Ario, Procedia Computer Science, 72, (2015), 217-224.

17. Y. Lin, Studies on the Characteristics of the Implantable Axial Blood Pump's Vibration Caused by Magnetic Force,(Changsha, Central South University, 2015).

18. Z. Yun, Y. Lin, C. Xiang, J. Huazhong Univ. of Sci. \& Tech. (Natural Science Edition), 41, 9(2013), 97101. 